INPLASY

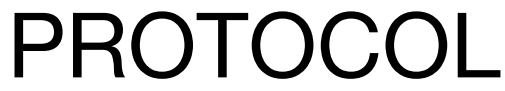

To cite: Bao et al. Clinical significance of plasma D-dimer and fibrinogen in outcomes after stroke: A Systematic Review and Meta-Analysis. Inplasy protocol 202210025. doi:

10.37766/inplasy2022.1.0025

Received: 06 January 2022

Published: 06 January 2022

Corresponding author:

Qiangji Bao

iloveyoucmu@163.com

Author Affiliation:

Qinghai Provincial People's

Hospital.

Support: No. 2021-wjzd-01.

Review Stage at time of this submission: Completed but not published.

Conflicts of interest:

None declared.

\section{Clinical significance of plasma D- dimer and fibrinogen in outcomes after stroke: A Systematic Review and Meta-Analysis}

Bao, Q1; Zhang, JN2; Wu, XT33; Zhao, K4; Guo, Y5; Yang, MF6; Du, XF7.

Review question / Objective: P: stroke patients I:biomakers level C: biomakers level O: Poor prognosis after stroke S:Cohort study and case-control ect.

Condition being studied: Background.In recent years, there is a growing literature on the prognostic significance of D-dimer, fibrinolysis in stroke. However, there has not been an overall analysis of adverse outcomes after stroke. Therefore, the objective of this study was to comprehensively assess the correlation between D-dimer, fibrinolysis and prognosis in stroke through meta-analysis. Methods. A systematic literature review in PubMed, EMBASE, Cochrane Library databases was performed to identify all studies. The association of D-dimer and fibrinolysis level with prognosis of stroke patients were expressed as Odds Ratio (OR) with $95 \%$ confidence intervals $(95 \% \mathrm{Cl})$. Results. 54 studies comprising 21473 stroke patients were included. OR for fibrinogen and D-dimer levels were 1.09 (95\%Cl:1.00-1.20) and $1.18(95 \% \mathrm{Cl}: 0.43-3.23)$ for thrombus formation after stroke, $2.21(95 \% \mathrm{Cl}: 1.55-3.16)$ and $1.47(95 \% \mathrm{Cl}$ $: 1.31-1.65)$ for death after stroke, $1.65(95 \% \mathrm{Cl}: 1.49-1.82)$ and $1.69(95 \% \mathrm{Cl}: 1.40-2.03)$ for poor outcome. Fibrinogen has also been found to be an effective marker for predicting the risk of recurrence1.28 (95\% Cl:1.05-1.55), cardiovascular disease 1.44 (95\% Cl: 1.26-1.65), and cognitive impairment1.87(95\% Cl: 1.24-2.81) in stroke patients. Conclusion. The level of fibrinogen and D-dimer was associated with adverse outcomes in patients with stroke. Both of them could be a potential prognostic marker in stroke.

INPLASY registration number: This protocol was registered with the International Platform of Registered Systematic Review and Meta-Analysis Protocols (INPLASY) on 06 January 2022 and was last updated on 06 January 2022 (registration number INPLASY202210025).

\section{INTRODUCTION}

Review question / Objective: P: stroke patients I:biomakers level C: biomakers level O: Poor prognosis after stroke S:Cohort study and case-control ect. 
Condition being studied: Background.In recent years, there is a growing literature on the prognostic significance of D-dimer, fibrinolysis in stroke. However, there has not been an overall analysis of adverse outcomes after stroke. Therefore, the objective of this study was to comprehensively assess the correlation between D-dimer, fibrinolysis and prognosis in stroke through metaanalysis.Methods. A systematic literature review in PubMed, EMBASE, Cochrane Library databases was performed to identify all studies. The association of Ddimer and fibrinolysis level with prognosis of stroke patients were expressed as Odds Ratio (OR) with $95 \%$ confidence intervals $(95 \% \mathrm{Cl})$. Results. 54 studies comprising 21473 stroke patients were included. OR for fibrinogen and D-dimer levels were 1.09(95\% Cl:1.00-1.20) and 1.18 (95\% Cl: 0.43-3.23) for thrombus formation after stroke, $2.21(95 \% \mathrm{CI}: 1.55-3.16)$ and $1.47(95 \% \mathrm{Cl}: 1.31-1.65)$ for death after stroke, $1.65(95 \% \mathrm{Cl}: 1.49-1.82)$ and $1.69(95 \% \mathrm{Cl}: 1.40-2.03)$ for poor outcome. Fibrinogen has also been found to be an effective marker for predicting the risk of recurrence 1.28(95\% CI:1.05-1.55), cardiovascular disease $1.44(95 \% \mathrm{Cl}$ : 1.26-1.65), and cognitive impairment 1.87(95\% Cl: 1.24-2.81) in stroke patients. Conclusion. The level of fibrinogen and Ddimer was associated with adverse outcomes in patients with stroke. Both of them could be a potential prognostic marker in stroke.

\section{METHODS}

Search strategy: This systematic review identified all studies on two markers in the stroke population. Three databases (PubMed, EMBASE, and Cochrane) were searched systematically for studies published prior to November 2021 by 2 independent researchers using identical search terms. Used terms were "D-dimer fibrin," "D-dimer," or "fibrin fragment DD" or "fibrin fragment D-dimer" or "D-dimer fragments" or "Fibrinogen" or "Coagulation Factor I" or "Factor I" or "gamma Fibrinogen" and "Stroke" or "Cerebrovascular*" and "Humans." The specific details of the search strategies for the three databases had been provided in Table 1. To prevent missing studies that did not have "plasma biomarker" as a key word, we also hand-searched the reference lists of all included studies and relevant review articles. When relevant information was unavailable, efforts were made to contact corresponding authors. Both researchers conducted qualification assessments. Inclusion and exclusion criteria were used to screen eligible studies by title and abstract.

Participant or population: Stroke patients.

Intervention: Biomaker level.

\section{Comparator: Biomaker level.}

Study designs to be included: Study inclusion criteria were as follows: 1 . Studies reporting the relationship of 2 biomarkers with stroke outcome; 2. Samples were taken from patients who had suffered a stroke; 3. Studies assessing personal levels of biomarkers (e.g., Ddimer and fibrinogen levels in blood); 4. Studies focused on human data; 5 . Studies published in English.

Eligibility criteria: Study exclusion criteria were as follows:1. Studies that only reported mean levels and standard deviations of biomakers in cases and noncases; 2. Studies without quantitative biomakers level data; 3 . Studies without original data; 4. Study type of review; 5. Study type of case report; 6 . Study type of comment.

Information sources: Electronic databases.

Main outcome(s): In conclusion, our study provides strong evidence that fibrinogen and D-dimer level was associated with adverse outcomes in patients with stroke. Fibrinogen and D-dimer level could be a potential prognostic marker in stroke. Combining the two biomarkers in clinical follow-up therapy may help customize treatment and improve the effectiveness of current stroke treatment strategies. 
Quality assessment / Risk of bias analysis: Two independent reviewers conducted the search, selection of studies, data extraction, and assessment of quality. Disagreements were resolved with a third reviewer. The study data were abstracted independently and were classified by biomarkers. Categorized data consisted of author's name and publication year, location of investigation, study design, sample size, study period, fibrinogen cutoff and D-dimer cutoff, Pooled OR $(95 \% \mathrm{Cl})$, outcomes, follow-up duration, variables adjusted for,ect. The Risk Of Bias In Nonrandomised Studies of Interventions (ROBINS-I) was used to performe risk of bias appraisal for included studies. All studies were scored on the following topics: Confounding bias, Selection bias, Deviation from intended intervention, Missing data, Measurement in outcome, Selection of reported result, Classification of intervention. In addition, the Oxford Centre for Evidence Based Medicine tool were used to assess the overall level of evidence presented in the literature.

Strategy of data synthesis: We performed the analysis with STATA 16.0 (Stata, version16.1; college Station, Texas 77845 USA). The study was a meta-analysis based on a random-effects model. We extracted cohort-specific ORs from Cox hazard models. In fibrinogen primary analysis, ORs and $95 \% \mathrm{Cl}$ were calculated for overall post-stroke thrombosis, adverse functional outcome, death, stroke recurrence, and other complications. Thrombosis, adverse functional outcomes, death, and neurological complications after stroke were calculated in the D-dimer analysis. When there were two or more studies, subgroup analysis of fibrinogen and Ddimer were performed for follow-up time and classification of complications. Publication bias was assessed by funnel plot in stata 16.0. We used the "metainf" and "forest" functions in the "meta" package to search for potential outliers in $\mathbf{R}$ for sensitivity analysis by omitting each study or specific study. Heterogeneity between studies was determined by Cochran's $\mathbf{Q}$ test and Higgins I squared. $\mathbf{P}<$ 0.1 or $12>50 \%$ was considered as heterogeneity. All $P$ values less than 0.05 on two-sided were considered statistically significant.

Subgroup analysis: When there were two or more studies, subgroup analysis of fibrinogen and D-dimer were performed for follow-up time and classification of complications.

Sensitivity analysis: No.

Language: English.

Country(ies) involved: China.

Keywords: D-dimer, Fibrinogen, Stroke, Prognostic, Meta-analysis.

Contributions of each author:

Author 1 - Qiangji Bao.

Author 2 - Jing-Ni Zhang.

Author 3 - Xin-Ting Wu.

Author 4 - Kai Zhao.

Author 5 - Yu Guo.

Author 6 - Mingfei Yang.

Author 7 - Xiao-Feng Du. 